\title{
SCOPING REVIEW SOBRE EL METAPLAN COMO METODOLOGÍA DE INVESTIGACIÓN CUALITATIVA EN CIENCIAS DE LA SALUD
}

\author{
Silvia Veiga-Seijo ${ }^{1}$, Maria Jesús Movilla-Fernández ${ }^{2}$ y Natalia Rivas-Quarneti ${ }^{3}$ \\ 1,2 y 3 Universidade da Coruña, España. ${ }^{1}$ silvia.veiga.seijo@udc.es, ${ }^{2}$ maria.jesus.movilla@udc.es e \\ ${ }^{3}$ natalia.rivas.quarneti@udc.es
}

\begin{abstract}
Resumen. Introducción: La Investigación Cualitativa Crítica en Ciencias de la Salud presenta un gran valor para generar conocimiento sobre la salud como fenómeno complejo. Desde este posicionamiento teórico, el Metaplan es un método de recolección de datos clave, cuyo uso se ha ampliado en los últimos años en las Ciencias Sociosanitarias. Objetivo: Explorar el alcance de la producción científica con respecto al Metaplan como herramienta empleada en estudios del ámbito de Ciencias de la Salud. Métodos: Se ha llevado a cabo una revisión de alcance de tipo scoping review siguiendo el marco metodológico de Arskey \& O'Malley en 2017. Se consultaron bases de datos multidisciplinares y del ámbito de Ciencias de la Salud, estableciéndose criterios de selección específicos. Se empleó un análisis temático. Resultados: los resultados describen el Metaplan en Ciencias de la salud, sus usos, características, potencialidades y tendencias en la investigación. Conclusiones: El Metaplan es una potencial herramienta para ser empleada en estudios que aborden la salud y el bienestar de las poblaciones desde una mirada crítica. Además, facilita la acción dialógica y procesos colaborativos-participativos que persiguen la transformación social.
\end{abstract}

Palabras clave: Metaplan; Investigación Cualitativa Crítica en Ciencias de la Salud; Salud.

\section{SCOPING REVIEW ABOUT METAPLAN AS A GROUP FACILITATION TECHNIQUE IN CRITICAL QUALITATIVE HEALTH RESEARCH}

\begin{abstract}
Introduction: Critical Qualitative Health Research has a great value in generating knowledge about health as a complex phenomenon. From this theoretical position, Metaplan is a key data collection method, and its use has been expanded in recent years in the Health Sciences field. Goals: To explore the scope of literature regarding the Metaplan as a tool employed in studies in the field of Health Sciences. Methods: A scoping review was conducted adopting Arskey \& O'Malley's methodological framework in 2017. Multidisciplinary and Health Sciences databases were consulted, establishing specific selection criteria. Articles were analyses using thematic analysis. Results: The results illustrate studies which employed Metaplan in the field of Health Sciences: its uses, characteristics, pontentialities, and research trends. Conclusions. The Metaplan is a potential tool to be used in studies that address the health and well-being of populations from a critical perspective. Furthermore, it facilitates the dialogical action and collaborative-participatory processes in order to foster social transformation
\end{abstract}

Keywords: Metaplan; Critical Qualitative Health Research; Health.

\section{INTRODUCCIÓN}

La complejidad del mundo requiere que en investigación existan múltiples enfoques para la generación de conocimiento (Hart, Poole, Facey, \& Parsons, 2017), lo que implica un debate paradigmático y plural. El modo en que los seres humanos otorgan sentido a sus experiencias de vida, determinadas por el contexto sociocultural, político y económico; y al mundo en el que viven, es el foco de la investigación cualitativa. Desde su desarrollo, se han generado múltiples enfoques y paradigmas dentro de su marco más amplio, motivo por el 
que la investigación cualitativa es conocida como un término "paraguas", ya que aglutina diversas orientaciones filosóficas, teóricas y metodológicas (Holloway \& Galvin, 2017).

En el ámbito de las Ciencias Sociosanitarias, el paradigma positivista ha presentado una elevada dominancia dentro de la práctica e investigación en salud, lo que se asocia con la expansión de la economía neoliberal del conocimiento (Kontos \& Grigorovich, 2018). No obstante, el interés en la Investigación Cualitativa en Salud se ha ampliado internacionalmente por ser una herramienta útil para estudiar fenómenos desde la perspectiva de las personas y profesionales; contribuyendo a la mejora de nuestra práctica profesional y a la práctica basada en la evidencia (Holloway \& Galvin, 2017).

Partiendo de este bagaje teórico, este trabajo se enmarca en el paradigma sociocrítico de la teoría crítica, la cual se caracteriza por su conciencia crítica, pues cuestiona la realidad, desafía el status quo e intenta producir cambio para alcanzar una sociedad más libre, justa y democrática (Sandín, 2003). En consonancia, este trabajo es aproximado desde la Investigación Cualitativa Crítica en Salud, la cual permite "hacer preguntas que van más allá de los supuestos y entendimientos prevalecientes, y reconoce el papel del poder y la posición social en los fenómenos relacionados con la salud" (Centre for Critical Qualitative Health Research, 2018). Como se observa, este tipo de investigación reconoce los elementos sociales, culturales, políticos y estructurales como determinantes de la salud, yendo más allá de la propia enfermedad y mirada individualista de un concepto de salud incipiente. Diferentes voces en el mundo han planteado la necesidad de argumentar y reafirmar el valor de la Investigación Cualitativa Crítica en Salud para generar conocimiento sobre la salud y bienestar de las personas (Hart et al., 2017) puesto que proporciona una "base sólida para un trabajo teórico y metodológico sólido con implicaciones prácticas, educativas y políticas" (p.1765).

La investigación cualitativa tiene a su alcance una gran variedad de métodos de generación de datos. Holloway \& Galvin (2017) establecen que la elección de la metodología y procedimientos dependen tanto de la pregunta de investigación, como de la postura epistemológica, las capacidades, habilidades y conocimiento del/a investigador/a y recursos disponibles para el proyecto. En investigación cualitativa, los métodos más ampliamente utilizados son entrevistas (en profundidad o semiestructuradas), observación participante, grupos focales o análisis de documentos. El uso de material multimedia como fotografías, grabaciones, vídeos y dispositivos están aumentando (Grove \& Gray, 2019). Así mismo, se 
ha ilustrado el uso de los mapas corporales narrados (Gastaldo, Rivas-Quarneti, Magalhães, 2018).

El planteamiento teórico hasta aquí desarrollado implica que como investigadoras/es pensemos y reflexionemos sobre los métodos de generación de datos en función de la pregunta de investigación y epistemología planteada. Desde la Investigación Cualitativa Crítica en Ciencias de la Salud, el diseño de generación de datos debe dirigirse a la transformación social de grupos silenciados, borrados o excluidos dentro de nuestras comunidades. En España, el trabajo de Rivas-Quarneti, Movilla-Fernández, \& Magalhães (2018), focalizada en vislumbrar acciones colaborativas de promoción de la salud en mujeres inmigrantes, describe una armazón teórica y metodológica situando el Metaplan como método coherente con la Investigación Cualitativa Crítica en Ciencias de la Salud y con el paradigma sociocrítico, ya que permite la acción dialógica y que el diseño sea realmente participativo. Este es un ejemplo de cómo articular métodos de recolección de datos relevantes que responda al objetivo del estudio desde una posición epistemológica crítica. En la literatura, investigaciones recientes señalaron el gran potencial del Metaplan en el ámbito de las Ciencias de la Salud (Olivar et al., 2015; Villar et al., 2016; Rivas-Quarneti, Movilla-Fernández, \& Magalhães, 2018).

\subsection{Pregunta de revisión y objetivo}

Debido al interés emergente en el Metaplan como técnica en investigación cualitativa, el presente trabajo plantea una revisión de alcance de la literatura con el fin de responder a las preguntas de investigación formuladas:

- ¿Es el Metaplan una técnica de facilitación en el ámbito de la investigación cualitativa en Ciencias de la Salud?

- ¿Cuáles han sido sus usos y en qué contextos? ¿cuáles son las características claves del Metaplan como elemento de facilitación para la investigación?

El objetivo es conocer el alcance y los temas de interés en la evidencia científica acerca del Metaplan como técnica en estudios de investigación cualitativa en el contexto de ciencias sociosanitarias. 


\section{METODOLOGÍA}

Para mapear y conocer el alcance de la producción científica respecto al Metaplan, se realizó una revisión de alcance inspirándose en la metodología "scoping review" propuesta por Arskey \& O’Malley (2005). Este tipo de metodología se usa para entender e interpretar de modo eficiente los temas y tendencias presentes en la literatura, así como identificar lagunas en la misma. La revisión se realizó entre enero y marzo del 2017.

Con el fin de localizar información científica relevante, se buscó en 12 bases de datos: a) bases de datos de revisiones sistemáticas: The Cochrane Library, b) multidisciplinares internacionales: Scopus, Web of Science, Dialnet y CSIC; c) específicas del ámbito de Ciencias de la Salud como Pubmed, Cinahl, Psycinfo, Psychological Database; y d) otras bases de datos del ámbito de la educación y economía como Busines, Enconlit y Eric. También se buscó en Google Académico y búsqueda a la inversa.

Los términos de búsqueda empleados en español fueron "Metaplan", "Investigación Cualitativa", "Investigación", "Métodos" y "Ciencias de la Salud", con sus respectivos en inglés. Dichos descriptores fueron consultados en el tesauro Descriptores en Ciencias de la Salud (DeCS).

Tras una búsqueda preliminar, observamos y analizamos que el uso del Metaplan en la investigación es incipiente y está cobrando cada vez más uso, pero los resultados son limitados. Por ello, para establecer la búsqueda bibliográfica, utilizamos como campo de búsqueda "Metaplan", ya que consiguió alcanzar un mayor número de resultados sin excluir material relevante. Los criterios de selección son los presentados en la Cuadro 1. Para el proceso de selección de los estudios se siguió la declaración PRISMA. El análisis de resultados se basó en un análisis temático (Braun \& Clarke, 2006).

Cuadro 1. Criterios de selección de los estudios en la revisión

\begin{tabular}{ll}
\hline Característica & Criterios de selección \\
\hline Tipo de estudio & Se incluyeron artículos originales, textos argumentativos, \\
& libros, capítulos de libro. \\
& Se excluyeron artículos de opinión. \\
Año de publicación & No se estableció criterios. \\
Idioma & Se han incluido artículos en español, inglés, gallego, \\
Temática & portugués y alemán. \\
& Focalizados en el Metaplan.
\end{tabular}




\section{EL METAPLAN EN INVESTIGACIÓN CUALITATIVA: RESULTADOS DE LA REVISIÓN}

A continuación, se presenta un análisis preliminar de los resultados. Los años de publicación de los estudios abarcan entre 1979 y 2016 . En la literatura, los términos empleados para hacer alusión al Metaplan son: meta-plan, meta-planning, metaplanning, metaplan-method, metaplán, metaplan-technique, metaplantechnique, metaplans, meta-plans, M-etaplán, Metaplan®.

\section{1 ¿Qué es el Metaplan y en qué consiste?}

El Metaplan es una herramienta de investigación cualitativa (Rivas-Quarneti, Magalhaes, \& Movilla-Fernández, 2017) como metodología participativa (Magalhães, Farias, RivasQuarneti, Alvarez, \& Malfitano, 2018). Se define como una técnica de resolución de problemas con base en la cooperación (Schnelle, 1979) y de facilitación grupal, que requiere la participación de todos los actores implicados "para la búsqueda colaborativa de mejoras o soluciones a una situación común a través de la visualización permanente de la discusión" (Rivas-Quarneti, Movilla-Fernández, \& Magalhães, 2018, p.677). También es definido como un brainstorming estructurado, como en el de Oliva, Brosa, Espín, Figureras, \& Trapero (2015) o como método de resolución de problemas. Las sesiones de Metaplan son diseñadas y moderadas por un facilitador cuyo fin es guiar la discusión o debate promoviendo una atmosfera de comunicación libre con el fin de lograr el objetivo establecido. Para ello, se establecen normas y estructura comunes para generar consenso. La sesión consiste en el uso de tarjetas de diferentes colores y formas sobre las que se fomenta la interacción y un flujo constante de discusión. Dichas tarjetas se irán colocando en paneles a la vista de las personas participantes. El/la facilitador/a debe asegurarse que todas las personas tengan oportunidad de participar (Valverde, Vidal, \& Jamsá, 2012; RivasQuarneti, 2015; Rivas-Quarneti, Magalhaes, \& Movilla-Fernández, 2017; Veiga-Seijo, 2017). El material empleado puede variar: panel, tarjetas o adhesivos de diferentes colores y formas, chinchetas/pegamento o celo y rotuladores de colores (Rivas-Quarneti, Magalhaes, \& Movilla-Fernández, 2017). El Metaplan debe hacerse en grupos pequeños, siendo el número adecuado entre 5 y 10 (Valverde, Vidal, \& Jamsá, 2012), o 8-10 (Olivares et al., 2015). 


\subsection{Etapas del Metaplan}

Las fases del Metaplan se explican de forma detallada en la Figura 1, inspiradas en diferentes investigaciones (Schnelle, 1979; Hughes, Saadi, Madoz, \& González, 2009; Valverde, Vidal, \& Jamsá, 2012; Miravitlles, 2015; Oliva et al., 2015; Olivares et al., 2015; Villar et al., 2016; Rivas-Quarneti, 2015; Rivas-Quarneti, Magalhaes, \& Movilla-Fernández, 2017; Veiga-Seijo, 2017):

Etapas del Metaplan

\begin{tabular}{|c|c|c|c|c|}
\hline Paso 1 & Paso 2 & Paso 3 & Paso 4 & Paso 5 \\
\hline $\begin{array}{l}\text { - Presentación } \\
\text { moderador y } \\
\text { participantes. } \\
\text { Exposición de } \\
\text { sesión: normas, } \\
\text { materiales y } \\
\text { tiempos. } \\
\text { - Se presenta el } \\
\text { panel de discusión } \\
\text { sobre el que se } \\
\text { trabajará en el } \\
\text { Metaplan. }\end{array}$ & $\begin{array}{l}\text { - Facilitador/a } \\
\text { introduce } \\
\text { pregunta de } \\
\text { investigación en } \\
\text { el panel } \\
\text { visualizado por } \\
\text { todos/as los/as } \\
\text { participantes, a } \\
\text { quienes se les } \\
\text { reparten tarjetas } \\
\text { de diferentes } \\
\text { tamaños y } \\
\text { colores. }\end{array}$ & $\begin{array}{l}\text { - Cada participante } \\
\text { esribe en su } \\
\text { tarjeta, en base a } \\
\text { sus creencias y } \\
\text { reflexiones, sobre } \\
\text { la pregunta de } \\
\text { estudio. La letra } \\
\text { debe ser grande y } \\
\text { clara para ser } \\
\text { visualizada } \\
\text { fácilmente por el } \\
\text { grupo. }\end{array}$ & $\begin{array}{l}\text { - Facilitador/a } \\
\text { recoge y baraja } \\
\text { las tarjetas. Las } \\
\text { lee en voz alta y } \\
\text { coloca en el } \\
\text { panel. Se agrupan } \\
\text { por contenido o } \\
\text { similitud con } \\
\text { ayuda de } \\
\text { participantes } \\
\text { (consenso) y se } \\
\text { abre discusión } \\
\text { sobre cada } \\
\text { tarjeta. }\end{array}$ & $\begin{array}{l}\text { - Se asigna un título } \\
\text { a cada grupo de } \\
\text { tarjetas. } \\
\text { - Se genera mapa } \\
\text { conceptual o } \\
\text { "nube de ideas". } \\
\text { Se anima a } \\
\text { participar e } \\
\text { interactuar, } \\
\text { añadiendo nuevas } \\
\text { contriuciones. } \\
\text {-Se realiza } \\
\text { resumen del } \\
\text { panel. }\end{array}$ \\
\hline
\end{tabular}

Figura 1. Etapas del Metaplan

\subsection{Ventajas e inconvenientes del Metaplan}

Las ventajas e inconvenientes encontrados en la literatura se presentan analizados en la Cuadro 2 (Rivas-Quarneti, Magalhães, \& Fernández, 2017; Veiga-Seijo, 2017; Miravitlles, 2015; Magalhães et al., 2019).

Cuadro 2. Ventajas e Inconvenientes del Metaplan

\begin{tabular}{|c|c|}
\hline Ventajas & Inconvenientes \\
\hline $\begin{array}{l}\text { - Favorece participación igualitaria entre participantes. } \\
\text { - Permite visualizar el trabajo realizado en conjunto. } \\
\text { - Facilita un debate ordenado para determinar puntos de consenso y } \\
\text { disentimiento. } \\
\text { - Permite llegar a acuerdos democráticamente. } \\
\text { - Apoya proceso de elevar conciencia y co-creación de alternativas o } \\
\text { soluciones. } \\
\text { - Garantiza anonimato. } \\
\text { - Constancia de los datos mediante las tarjetas. } \\
\text { - Permite organización eficaz de los resultados y análisis colaborativo e } \\
\text { in situ. } \\
\text { - Proporciona fiabilidad y validez, objetividad } \\
\text { - Eficacia y optimización del tiempo. Bajo coste. }\end{array}$ & $\begin{array}{l}\text { - Formación y práctica en } \\
\text { la técnica para tener un } \\
\text { control de la sesión. } \\
\text { El/la facilitador/a debe } \\
\text { ser consciente de su rol } \\
\text { como investigador } \\
\text { evitando juicios de } \\
\text { valor. }\end{array}$ \\
\hline
\end{tabular}




\subsection{Usos del Metaplan en Ciencias de la Salud}

En gran parte de los estudios incluidos, la población de estudio son profesionales de las ciencias sociosanitarias, como es el caso de Magalhães et al. (2019). Estudios como el de Rivas-Quarneti (2015) se focalizan en personas o poblaciones. Se encontraron estudios que usaron el Metaplan para:

- Identificar intervenciones por parte de profesionales en cuestión de costo-efectividad.

- Establecer consenso sobre recomendaciones para informar o crear guías (Olivares et al., 2015), recoger propuestas; o para discutir temas identificados en la literatura.

- Conocer las opiniones, actitudes y conocimientos con relación a procesos saludenfermedad Miravitlles et al, 2015) así como evaluar o identificar necesidades, problemas y demandas.

\section{CONCLUSIONES}

El Metaplan presenta un gran potencial como método de investigación cualitativo participativo en salud, pues optimiza la discusión equitativamente, promueve la reflexividad crítica y la creación de alternativas. Es necesario desarrollar más investigaciones cualitativas empleando el Metaplan para profundizar en sus contribuciones a la Investigación Cualitativa en Ciencias de la Salud.

\section{REFERENCIAS}

Álvarez, F. V., Zabaleta, R. M., Solves, J. M., Corbella, E. C., Lobato, S. D., González-Torralba, F., ... \& Lozano11, P. V. (2016). Qué no hacer en la EPOC. Análisis metodológico. Revista de Patología Respiratoria, 19(3).

Arksey, H., \& O'Malley, L. (2005). Scoping studies: towards a methodological framework. International journal of social research methodology, 8(1), 19-32.

Braun, V., \& Clarke, V. (2006). Using thematic analysis in psychology. Qualitative research in psychology, 3(2), 77-101.

Centre for Critical Qualitative Health Research, Facey, M., Gastaldo, D., Gladstone, B., \& Gagnon, M. (2018). Learning and Teaching Qualitative Research in Ontario: A Resource Guide. Toronto.

Gastaldo, D., Rivas-Quarneti, N., \& Magalhães, L. (2018). Body-map storytelling as a health research methodology: blurred lines creating clear pictures. Forum Qualitative Social Research, 19(2).

Grove, S. K. \& Gray, J. R. (2019). Investigación en enfermería: Desarrollo de la práctica enfermera basada en la evidencia. Barcelona: Elsevier.

Hart, C., Poole, J. M., Facey, M. E., \& Parsons, J. A. (2017). Holding firm: Power, push-back, and opportunities in navigating the liminal space of critical qualitative health research. Qualitative health research, 27(12), 17651774. 
Holloway, I., \& Galvin, K. (2017). Qualitative Research in Nursing and Healthcare. Chichester: Wiley Blackwell.

Hughes, D., Saadi, M. F., Madoz, M. C., \& González, A. H. (2009). Aplicación para la administración y desarrollo de cursos con la técnica de Metaplan que aporta etapas virtuales mediante la Web. In XV Congreso Argentino de Ciencias de la Computación.

Kontos, P., \& Grigorovich, A. (2018). " Sleight of Hand" or" Selling Our Soul"? Surviving and Thriving as Critical Qualitative Health Researchers in a Positivist World. Forum Qualitative Social Research, 19(2). http://dx.doi.org/10.17169/fqs-19.2.2990

Magalhães, L., Farias, L., Rivas-Quarneti, N., Alvarez, L., \& Malfitano, A. P. S. (2019). The development of occupational science outside the Anglophone sphere: Enacting global collaboration. Journal of Occupational Science, 26(2), 181-192.

Miravitlles, M., Alcázar, B., Alvarez, F. J., Bazús, T., Calle, M., Casanova, C., ... \& García-Sidro, P. (2015). What pulmonologists think about the asthma-COPD overlap syndrome. International journal of chronic obstructive pulmonary disease, 10, 1321. http://dx.doi.org/10.2147/COPD.S88667

Oliva, J., Brosa, M., Espín, J., Figueras, M., \& Trapero, M. (2015). Cuestiones controvertidas en evaluación económica (I): perspectiva y costes de intervenciones sanitarias. Revista Española de salud Pública, 89(1), 5-14.

Olivares Cortés, S., Zacarías Hasbún, I., González González, C. G., Fonseca Morán, L., Mediano Stoltze, F., Pinheiro Fernandes, A. C., \& Rodríguez Osiac, L. (2015). Diseño y validación de la imagen para la difusión e implementación de las guías alimentarias para la población chilena. Nutrición Hospitalaria, 32(2), 582589.

Rivas-Quarneti, N. (2015). Estudio De las ocupaciones cotidianas para la promoción de la salud de las mujeres inmigrantes en situación de vulnerabilidad. Universidade da Coruña.

Rivas-Quarneti, N., Magalhaes, L., \& Fernández, M. (2017). Contribuciones del Metaplan en Investigación Cualitativa Crítica en Ciencias de la Salud: un camino hacia la acción dialógica. CIAIQ, 2. Disponible en: https://proceedings.ciaiq.org/index.php/ciaiq2017/article/view/1262/1222

Rivas-Quarneti, N., Movilla-Fernández, M. J., \& Magalhães, L. (2018). Immigrant women's occupational struggles during the socioeconomic crisis in Spain: broadening occupational justice conceptualization. Journal of Occupational Science, 25(1), 6-18.

Sandín, MP. (2003). Investigación cualitativa en educación. Fundamentos y tradiciones. Madrid: Editorial Mcgraw.

Schnelle, E. (1979). The Metaplan-method: Communication Tools for Planning and Learning Groups. Metaplan$\mathrm{GmbH}$.

Valverde, M., Vidal, M., \& Jansá, M. (2012). Técnicas didácticas en educación terapéutica aplicadas a la diabetes. Revista Rol de Enfermería, 35(10), 682-691.

Veiga-Seijo, S. (2017). Estudio cualitativo sobre el baremo de valoración de la situación de dependencia en Galicia: ventajas, retos y propuestas de mejora desde la perspectiva de los valoradores. Universidade da Coruña. Disponible en: https://ruc.udc.es/dspace/handle/2183/23138

Villar Álvarez, F., Moreno Zabaleta, R., Mira Solves, J.J., Calvo Corbella, E., Díaz Lobato, S., González-Torralba, F.,...Navarro Soler, I. (2016). Qué no hacer en la EPOC. Análisis metodológico. Revista de Patología Respiratoria, 19(3): 76-82. 